https://helda.helsinki.fi

\title{
Missense variant in LOXHD1 is associated with canine nonsyndromic hearing loss
}

\section{Hytönen, Marjo K.}

$2021-11$

Hytönen , M K , Niskanen , J E , Arumilli , M , Brookhart-Knox , C A , Donner , J \& Lohi , H 2021 , ' Missense variant in LOXHD1 is associated with canine nonsyndromic hearing loss ', Human Genetics , vol. 140 , pp. 1611-1618 . https://doi.org/10.1007/s00439-021-02286-z

http://hdl.handle.net/10138/335460

https://doi.org/10.1007/s00439-021-02286-z

cc_by

publishedVersion

Downloaded from Helda, University of Helsinki institutional repository.

This is an electronic reprint of the original article.

This reprint may differ from the original in pagination and typographic detail.

Please cite the original version. 


\title{
Missense variant in LOXHD1 is associated with canine nonsyndromic hearing loss
}

\author{
Marjo K. Hytönen ${ }^{1,2,3} \cdot$ Julia E. Niskanen ${ }^{1,2,3} \cdot$ Meharji Arumilli1,2,3 $^{1}$ Casey A. Brookhart-Knox ${ }^{5}$. Jonas Donner ${ }^{4}$. \\ Hannes Lohi ${ }^{1,2,3}$
}

Received: 3 March 2021 / Accepted: 20 April 2021 / Published online: 13 May 2021

(c) The Author(s) 2021

\begin{abstract}
Hearing loss is a common sensory deficit in both humans and dogs. In canines, the genetic basis is largely unknown, as genetic variants have only been identified for a syndromic form of hearing impairment. We observed a congenital or early-onset sensorineural hearing loss in a Rottweiler litter. Assuming an autosomal recessive inheritance, we used a combined approach of homozygosity mapping and genome sequencing to dissect the genetic background of the disorder. We identified a fully segregating missense variant in $L O X H D 1$, a gene that is known to be essential for cochlear hair cell function and associated with nonsyndromic hearing loss in humans and mice. The canine LOXHDl variant was specific to the Rottweiler breed in our study cohorts of pure-bred dogs. However, it also was present in some mixed-breed dogs, of which the majority showed Rottweiler ancestry. Low allele frequencies in these populations, $2.6 \%$ and $0.04 \%$, indicate a rare variant. To summarize, our study describes the first genetic variant for canine nonsyndromic hearing loss, which is clinically and genetically similar to human $L O X H D 1$-related hearing disorder, and therefore, provides a new large animal model for hearing loss. Equally important, the affected breed will benefit from a genetic test to eradicate this $L O X H D 1$-related hearing disorder from the population.
\end{abstract}

\section{Introduction}

Hearing loss (HL) is the most common sensory impairment and consequently constitutes a major medical issue, affecting 1-3/1000 newborns and becoming more prevalent by age (Morton and Nance 2006). It is a both clinically and genetically heterogeneous disorder: the severity of the hearing impairment varies and the age of onset ranges from congenital and early-onset to late-onset. Other symptoms may accompany hearing impairment, but nonsyndromic hearing loss (NSHL) is the predominant type. Genetic factors contribute to more than half of the congenital and early childhood nonsyndromic forms, and variants have been reported

Hannes Lohi

hannes.lohi@helsinki.fi

1 Department of Medical and Clinical Genetics, University of Helsinki, Helsinki, Finland

2 Folkhälsan Research Center, Helsinki, Finland

3 Department of Veterinary Biosciences, University of Helsinki, Helsinki, Finland

4 Genoscoper Laboratories, Helsinki, Finland

5 Wisdom Health, Vancouver, WA, USA in over 100 genes to date (https://hereditaryhearingloss. org/). Despite the accumulating knowledge, a large number of patients remain without a molecular diagnosis. Thus, there is a need to better understand the molecular underpinnings of hearing loss and the associated genes and variants in cochlear biology and disease pathophysiology.

The molecular genetics of hearing loss can be addressed by studying spontaneous hearing defects in purebred dogs. Over the past century, selective breeding of dogs has led to hundreds of breeds with specific genomic architectures and the formation of genetic isolates, which can significantly facilitate gene discovery in small study cohorts (LindbladToh et al. 2005). Both syndromic and nonsyndromic congenital as well as adult-onset hearing loss have been reported in dogs across breeds (Strain. 2015), yet their genetic background remains mostly uncharacterized. The most common form of hearing loss in dogs is pigment-associated congenital sensorineural deafness, which occurs in breeds with a lack of pigmentation or piebaldism (De Risio et al. 2016; Comito et al. 2012; Platt et al. 2006; Strain 2004). The trait is associated with regulatory variants in melanocyte inducing transcription factor (MITF) (Karlsson et al. 2007). Another syndromic type of $\mathrm{HL}$ is a recessive congenital deafness and vestibular syndrome observed in Doberman Pinschers, 
which is linked to variants in myosin VIIA (MYOA7) and tyrosine phosphatase, receptor type Q (PTPRQ) (Webb et al. 2019; Guevar et al. 2018). In addition to the congenital HL forms, an adult-onset hearing defect has been reported in Border Collies and mapped to canine chromosome 6 (Yokoyama et al. 2012). In our study, we observed sensorineural hearing loss in Rottweilers and utilized genome-wide array genotyping and genome sequencing to identify a fully segregating novel missense variant in $L O X H D 1$, a known hearing loss gene in human.

\section{Materials and methods}

\section{Study cohorts}

EDTA blood samples were collected from 585 privately owned Rottweiler dogs, including four affected littermates. The samples were stored at $-20^{\circ} \mathrm{C}$ until genomic DNA was extracted using a semi-automated Chemagen extraction robot (PerkinElmer Chemagen Technologie GmbH). DNA concentration was determined either with NanoDrop ND-1000 UV/Vis Spectrophotometer or Qubit 3.0 Fluorometer (Thermo Fisher Scientific Inc.). Sample collection was ethically approved by the Animal Ethics Committee of State Provincial Office of Southern Finland (ESAVI/343/04.10. 07/2016 and ESAVI/25696/2020).

\section{Homozygosity mapping}

Four affected littermates, one unaffected littermate and two unrelated unaffected Rottweilers were genotyped using the CanineHD Whole-Genome Genotyping BeadChip containing 173,662 markers (Lincoln, NE, USA). Pre-analytical QC was conducted using PLINK (version 1.96b6.20, Chang et al. 2015) and included pruning for sample call rate of $>95 \%$, marker call rate of $>95 \%$, and Hardy-Weinberg equilibrium $\mathrm{p}$ value $<1 \times 10^{-8}$. One of the cases was discarded due to a poor call rate. As recommended by Meyermans et al. (2020), pruning for minor allele frequency or LD was not performed. After QC, three cases and three controls, as well as 154,235 markers remained for analysis.

Detection of runs of homozygosity ( $\mathrm{ROH})$ was also performed with PLINK 1.9 (Chang et al. 2015). Two rounds of analyses were conducted: first, the optimization of population-dependent parameters using simulated data; and second, detection of ROH shared by the affected dogs. In both analyses, the minimum marker size for ROH (--homozyg-snp) was set to 70 based on the formula described by Purfield et al. (2012) with $\alpha=0.05, N_{\mathrm{s}}=154,235, N_{\mathrm{i}}=6$ and mean SNP heterozygosity $=0.21$ as evaluated using an in-house Python script. Furthermore, the parameters --homozyg-window-snp,
Table 1 Values used for parameter optimization and detection of shared ROH in PLINK

\begin{tabular}{lll}
\hline Parameter & \multicolumn{2}{l}{ Values by analysis type } \\
\cline { 2 - 3 } & Parameter optimization & $\begin{array}{l}\text { Detection of } \\
\text { shared ROH }\end{array}$ \\
\hline $\begin{array}{l}\text {--homozyg-window-snp } \\
\text {--homozyg-window- } \\
\text { missing }\end{array}$ & 20 & 50 \\
--homozyg-window-het & 0 & 1 \\
--homozyg-window- & 0.05 & 1 \\
threshold & & 0.05 \\
--homozyg-snp & 70 & 70 \\
--homozyg-kb & $1000 \mathrm{~kb}$ & $1000 \mathrm{~kb}$ \\
--homozyg-gap & $2000 \mathrm{~kb}$ (when unvaried) & $200 \mathrm{~kb}$ \\
& $20-1000 \mathrm{~kb}$ (when varied) \\
--homozyg-density & $\begin{array}{l}200 \mathrm{~kb} / \mathrm{snp} \text { (when unvar- } \\
\text { ied) }\end{array}$ & $30 \mathrm{~kb} / \mathrm{snp}$ \\
& $10-125 \mathrm{~kb} / \mathrm{snp}$ (when \\
\multicolumn{2}{c}{ varied) } \\
\hline
\end{tabular}

--homozyg-window-missing, --homozyg-window-het, --homozyg-window-threshold and --homozyg-kb were set to a fixed value depending on the analysis (Table 1).

Finally, to establish suitable values for ROH density and maximum gap, genotypes for a fully homozygous individual were simulated based on the population map file and analyzed for maximal genome coverage as described by Meyermans et al. (2020). To calculate maximum genome coverage for the simulated genome, --homozyg-gap was set to $2000 \mathrm{~kb}$ and --homosyg-density to $200 \mathrm{~kb} / \mathrm{snp}$; genome coverage was determined as the total length of the resulting $\mathrm{ROH}$. The simulated genome was then analyzed by varying --homozyg-density from 10 to $125 \mathrm{~kb} / \mathrm{snp}$ in increments of $5 \mathrm{~kb}$ and --homozyg-gap from 20 to $1000 \mathrm{~kb}$ in increments of $20 \mathrm{~kb}$ (Table 1).

Based on the simulation, density was set to $30 \mathrm{~kb} / \mathrm{snp}$ and maximum gap to $200 \mathrm{~kb}$, as genome coverage reached $100 \%$ for --homozyg-density at 30 and increased only negligibly for --homozyg-gap after 200. Using these parameters, detection of ROH was performed with --homozyg-group. ROH included in further analyses were required to be allelically shared by all three cases and either allelically different or absent in the controls.

\section{Whole-exome and -genome sequencing}

Whole-exome sequencing (WES) was carried out for one affected dog of the initially identified Rottweiler litter. The exome library was prepared with 140702_canFam3_exomeplus_BB_EZ_HX1 kit with a capture size of $152 \mathrm{Mb}$ from the Roche NimbleGen SeqCap EZ target enrichment design (Broeckx et al. 2015). The library was sequenced with the 
Illumina NextSeq500 platform with a read length of $300 \mathrm{bp}$ (paired-end reads, $2 \times 150 \mathrm{bp}$ ) and a coverage of $38 \times$ at the Biomedicum Functional Genomics Unit (University of Helsinki, Finland). The exome sequence data analysis, including quality control, mapping, alignment post-processing, single nucleotide variant calling and small indel calling, was performed as described previously (Hytönen et al. 2019). Whole-genome sequencing (WGS) was conducted on another affected Rottweiler from the same litter on Illumina HiSeq2000 high-throughput sequencing platform with a read length of $100 \mathrm{bp}$ (paired-end reads, $2 \times 100 \mathrm{bp}$ ) and a coverage of $15 \times$ at the University of Bern. Reads from the WGS sample were processed using SpeedSeq open-source software with bwa (v0.7.15) for alignment, SAMBLASTER for marking duplicate reads, sorting and BAM compression using Sambamba (Chiang et al. 2015).

For WGS sample, the variant calling of single nucleotide variants (SNVs) and small insertions and deletions (indels) was done using the HaplotypeCaller in gVCF mode (v3.7-0), which is combined with gVCFs from our cohort with combineGVCFs and joint genotyping was done by GenotypeGVCFs in Genome Analysis Tool Kit GATK version 4.1 (McKenna et al. 2010). Mobile Element Locator Tool (MELT) was used to detect mobile element insertions (Gardner et al. 2017) and the reference sequences of the transposons for mobile element insertion (MEI) discovery were retrieved from the Repbase database (Jurka et al. 2005). The DELLY software was used to detect structural variants (SVs), including deletions, duplications, inversions and insertions by independent commands (Rausch et al. 2012). Functional annotation of variants from both WES and WGS samples was done using Ensembl release100 and NCBI Canis lupus familiaris Annotation Release 105.

The aligned bam files were submitted to SRA with the BioProject accession PRJNA702911. The sample accession for the exome sample is SAMN17983069 and SAMN17983068 for the WGS sample.

NCBI transcript XM_022421426.1 and UniProt sequence J9PAE4 were used to count the nucleotide and amino acid positions for LOXHD1.

\section{Variant analysis}

The identified variants were imported into a webGQT variant server deployed locally on in-house canine variant datasets for inheritance model-based candidate variant filtering (Arumilli et al. 2020). The variant data of the affected dogs were filtered against 637 control genomes (Online Resource 1) when filtering SNVs and indels, assuming an autosomal recessive inheritance of the disease and allowing a maximum of two heterozygous calls for each variant in the controls. The WGS data of one affected dog were filtered against 290 control genomes (Online Resource 1) when filtering for MEIs and SVs. For SNVs and indels, both cases were required to share the variants in the homozygous state; for SVs and MEIs, only the WGS case was used and both homozygous and heterozygous calls were included to allow for inaccuracies in calling. Prediction of the variant pathogenicity was assessed using PROVEAN (Choi and Chan 2015; Choi et al. 2012) and Poly-Phen2 software (Adzhubei et al. 2010). Finally, homology analysis of a candidate causal variant in the LOXHDI gene was performed by retrieving orthologous genes for XP_022277134.1 with NCBI's blastp web interface and aligning them with COBALT (Johnson et al. 2008; Papadopoulos and Agarwala 2007).

\section{Genomic DNA analysis}

Genotyping of individual dogs was performed with PCR followed by Sanger sequencing. The DNA template was amplified using a forward primer (5'-GCTGTGTGTTGGAGA AGCAA-3') and a reverse primer (5'-TAGTTGCCTGAC ACCCTGAG-3') flanking the LOXHDl variant with Taq polymerase (Biotools B\&M Labs, S.A.). The products were directly sequenced using the PCR primers on an ABI 3730 capillary sequencer (Life Technologies) after treatment with exonuclease I (New England Biolabs) and rapid alkaline phosphatase (Roche Diagnostics). The Sanger sequence data were analyzed using either Sequencher 5.4 (GeneCodes) or Unipro UGENE v1.32.0 (Rose et al. 2019; Okonechnikov et al. 2012; Golosova et al. 2014).

A sample of 28,116 dogs, including 374 different breeds (Online Resource 7), was submitted for commercial genetic testing at Genoscoper Laboratories (Wisdom Health Finland) during 2017-2020. Another study sample of 771,864 mixed-breed dogs was screened using Wisdom Panel ${ }^{\mathrm{TM}}$ (Wisdom Health, WA, USA) genetic testing, including breed detection assessment, during 2019-2021.

\section{Results}

Sensorineural bilateral deafness was diagnosed in four Rottweiler siblings (one female and three males) in a litter of ten puppies using brainstem auditory evoked response (BAER) testing. BAER testing was performed either at $4(n=2), 5$, or 19 months of age, and no auditory response was detected in any of them. However, owners' observations suggested that the puppies had already been affected by hearing impairment at a few weeks of age. No other clinical signs were observed.

We carried out a genome-wide analysis to identify candidate loci using three affected and three unaffected dogs. Homozygosity mapping resulted in 22 regions of case-specific, allelically matching runs of homozygosity (Fig. 1 and Online Resource 2). The regions spanned a total length of 


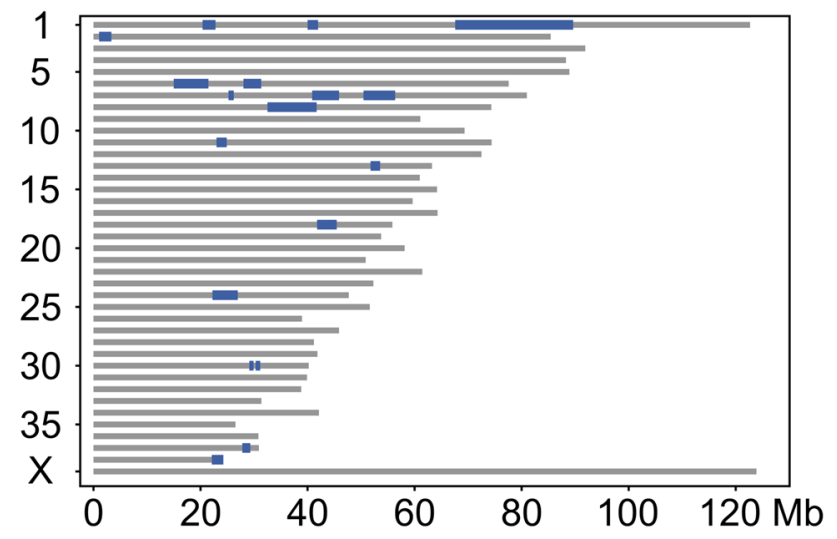

Fig. 1 Results of homozygosity mapping in three affected and three unaffected dogs. Case-specific, allelically matching ROH are indicated in blue
$62.3 \mathrm{Mb}$, the largest being $21.3 \mathrm{Mb}$ on chromosome 1 at $67,943,666-89,271,008$.

Subsequently, we performed whole exome sequencing on one affected dog and, later, whole genome sequencing on another case. As a result of variant filtering, 32 homozygous SNVs and indels shared by the two affected dogs were discovered; of these, six were located in case-specific $\mathrm{ROH}$ (Table 2, Online Resource 3). Second, 63 SVs and 32 MEIs called either homozygous or heterozygous and private to the sequenced affected dog were identified, and only one MEI resided in case-specific ROH (Table 2, Online Resource $4-5)$.

Of the seven case-specific variants that resided in $\mathrm{ROH}$, two exonic variants were considered for further analyses. First, a $\mathrm{G}>\mathrm{C}$ missense variant at chr7:44,806,821 in
Table 2 Case-specific SNVs and indels of two affected dogs (one WGS and one WES) and SVs and MEIs of one affected $\operatorname{dog}$ (WGS)

\begin{tabular}{|c|c|c|c|c|c|c|}
\hline \multirow[t]{2}{*}{ Variants } & \multicolumn{2}{|c|}{ SNVs and indels $(\mathrm{N})$} & \multicolumn{2}{|c|}{ SVs (N) } & \multicolumn{2}{|c|}{ MEIs (N) } \\
\hline & All & In $\mathrm{ROH}$ & All & In $\mathrm{ROH}$ & All & In $\mathrm{ROH}$ \\
\hline Total & 32 & 6 & 63 & 0 & 32 & 1 \\
\hline Exonic & 10 & 2 & 5 & 0 & 0 & 0 \\
\hline Intronic & 13 & 3 & 18 & 0 & 6 & 0 \\
\hline Splicing & 0 & 0 & 0 & 0 & 1 & 0 \\
\hline UTR & 4 & 1 & 1 & 0 & 0 & 0 \\
\hline Other & 5 & 0 & 39 & 0 & 25 & 1 \\
\hline
\end{tabular}

The variants were categorized according to NCBI Annotation Release 105

$S N V$ single nucleotide variant, indel small insertion or deletion, $S V$ structural variant, $M E I$ mobile element insertion, $\mathrm{ROH}$ runs of homozygosity

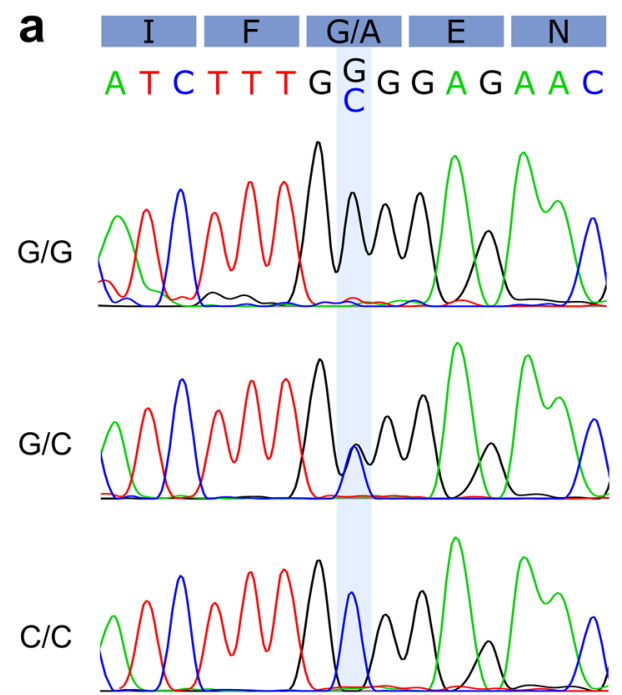

a

Fig. 2 a Example chromatograms from Sanger sequencing of the chr7:44,806,821G $>$ C variant. b Genotypes of four cases and three controls at a $\mathrm{ROH}$ at chr7:41.0-45.7 Mb. A distinct case-specific homozygous haplotype can be observed at $41.2-45.5 \mathrm{Mb}$. The bot-

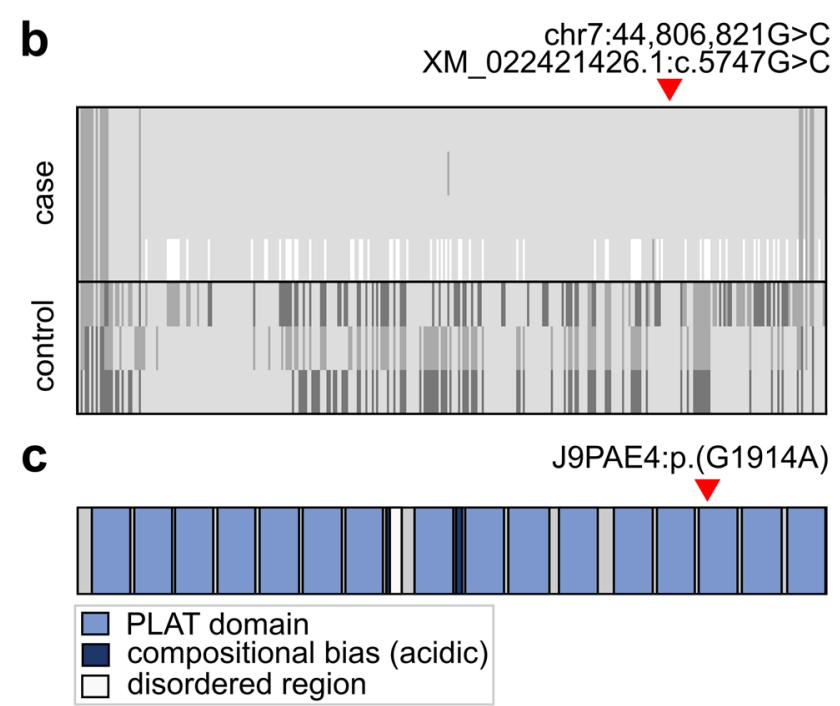

tommost case was not included in $\mathrm{ROH}$ detection due to a poor call rate. c Schematic illustration of the domain structure of LOXHD1 (J9PAE4) 
lipoxygenase homology domains 1 ( $L O X H D 1)$, a gene that is known to cause hearing loss in humans and mice (Grillet et al. 2009), was predicted to result in a glycine-to-alanine substitution (Fig. 2). Similarly, a $\mathrm{C}>\mathrm{T}$ missense variant at chr24:25,785,932 in maestro heat like repeat family member $8(M R O H 8)$ was predicted to cause a glycine-to-serine substitution. We assessed the pathogenicity of these variants in silico using two protein prediction tools, PROVEAN and PolyPhen-2. First, PROVEAN predicted both the LOXHDI and $M R O H 8$ substitutions as "deleterious" with a score of -4.517 and -3.336 , respectively. Similarly, PolyPhen-2 predicted the $L O X H D 1$ variant as "probably damaging" with a HumVar score of 0.992 and the MROH8 variant as "possibly damaging" with a score of 0.550 . As $M R O H 8$ has been associated with red blood cell volume, body mass index, telomere length and hippocampal atrophy in humans (Buniello et al. 2019) and its predicted impact was less pathogenic, making it an unlikely candidate variant, we focused on LOXHD1. The chr7:44,806,821G $>\mathrm{C}$ variant is predicted to cause a glycine-to-alanine substitution p.(G1914A) in the fourteenth PLAT domain of the canine LOXHD1 protein (Fig. 2c).

In addition, we assessed the conservation of the G1914 residue with multiple alignment of 99 Eutherian LOXHD1 protein orthologs, including the dog (Online Resource 6). In these species, the glycine residue and several flanking amino acids are fully conserved.

To validate the LOXHD1 variant, we genotyped it in a cohort of 585 Rottweilers, including the four affected siblings and 581 unaffected dogs. We observed complete segregation of the variant with hearing loss, as all four affected dogs were homozygous for the variant. The unaffected dogs were either heterozygous $(n=33)$ or wild-type $(n=548)$. The six unaffected littermates of the probands were either wild-type or heterozygous for the variant. The allele frequency in the population, excluding the affected family, was $2.6 \%$ and carrier frequency $5.3 \%$.

An additional sample of dogs submitted for commercial genetic testing was screened for the $L O X H D l$ variant to explore its distribution across breeds. All 28,116 tested dogs representing 374 breeds, breed varieties or designer dog mixes were found homozygous for the wild-type allele (Online Resource 7). Finally, the variant was also screened in a larger study sample of $771,864 \mathrm{dogs}$ submitted to genetic testing, including breed detection assessment. A variant carrier frequency of $0.08 \%$ and allele frequency of $0.04 \%$ were observed in this dataset. Interestingly, six dogs were found homozygous for the $L O X H D l$ variant. We were able to contact the owners of $4 / 6$ of the homozygous dogs and the owners reported profound hearing loss or deafness in all of them. One of the deaf dogs did not show any immediate Rottweiler ancestry, while one was a purebred Rottweiler and two were mixed-breed with Rottweiler ancestry.
Altogether, of the dogs carrying at least one copy of the deafness candidate variant, $63.4 \%$ showed evidence of Rottweiler ancestry in their immediate three-generation pedigree going back to great-grandparents, providing further support for a link between this specific breed background and the presence of the variant.

According to a study by Riazuddin et al. (2012), heterozygous LOXHD1 variants in humans have been suggested to contribute to autosomal dominant late-onset Fuchs endothelial corneal dystrophy (FECD). For this reason, we assessed the eye examinations performed as a part of the breeding programs and regular health check-ups of the Rottweilers genotyped either homozygous or heterozygous for the $L O X H D 1$ variant. Two of the homozygous deaf dogs had been eye examined healthy, one at 2 years and the other at 8 years old. In addition, eye examination reports were available for 22/33 heterozygous dogs, examined between 1 and 7 years and 4 months old. Three heterozygous dogs were diagnosed with different forms of cataract, which is a relatively common eye disease in Rottweilers. No signs of corneal dystrophy were reported in any of the dogs.

\section{Discussion}

We describe here a missense variant in $L O X H D 1$ associated with an autosomal recessive congenital nonsyndromic hearing loss in Rottweilers. The variant is rare, yet we confirmed it to be fully segregating with the disease in the breed. This is the first genetic defect identified for NSHL in dogs.

The dogs with the identified LOXHDI variant had either congenital or early-onset hearing loss. The owners' reports suggest that the puppies had at least some hearing impairment already at a few weeks of age. However, the confirmed diagnosis by BAER was acquired later and by that time, the hearing loss was total. Therefore, it is likely that the hearing impairment was congenital and progressed to deafness in a few months, although this remains unconfirmed. The hearing loss in Rottweilers can be defined as nonsyndromic as the affected dogs showed no other consistent clinical features.

Congenital deafness has been reported in Rottweilers previously as sporadic cases (Coppens et al. 2001; Strain 1996). Histopathological examination of one 4.5-monthold bilaterally deaf Rottweiler puppy demonstrated severe degeneration of hair cells and spiral ganglion while the vestibular organ was unaffected (Coppens et al. 2001). Strikingly similar changes are seen in the samba mouse line generated in an ethylnitrosourea (ENU) mutagenesis screen, where a Loxhdl missense variant leads to hearing loss (Grillet et al. 2009). Stereociliary development in samba mice is normal, but hair cell function is altered by postnatal day 21 and hair cells eventually undergo degeneration followed by possibly secondary loss of spiral 
ganglion neurons. Based on the similarities of the canine and murine models, it is probable that the histopathological changes described in the previous Rottweiler cases represent the canine $L O X H D 1$ c. $1914 \mathrm{G}>$ A variant identified in this study. It can be concluded that LOXHD1 has an essential role in maintaining normal cochlear hair cell function. Interestingly, murine Loxhdl is mainly expressed in the membrane of mature mechanosensory hair cells in the cochlea and high levels in testis, an organ also enriched with stereocilia. Its numerous PLAT (polycystin/lipoxygenase/alpha-toxin) domains are likely involved in targeting the protein to the plasma membrane (Grillet et al. 2009; Bateman and Sandford 1999), but the specific function of LOXHD1 in ciliary structures remains unclear.

In humans, several variants in LOXHDI have been reported to lead to autosomal recessive nonsyndromic hearing loss (ARNSHL) (DFNB77; OMIM \#613079) (Bai et al. 2020; Maekawa et al. 2019; Shen et al. 2019; Zhang et al. 2019; Mori et al. 2015; Riazuddin et al. 2012; Edvardson et al. 2011; Grillet et al. 2009). The severity of the patients' hearing impairment depends on the genetic defect and varies from mild to profound and is either stable or progressive. Moreover, the age of onset ranges from congenital to even adulthood. Interestingly, one of the human variants, p.(G1849R), is homologous in position with the canine p.(G1914A) substitution. The patient, who was compound heterozygous for LOXHDl with the p.(G1849R) and a p.(Y1541*) variant, had been diagnosed with severe congenital hearing loss at the age of two years (Plevova et al. 2017). The homology of the human and canine variant further suggests that substitution of the glycine residue interferes with the function of LOXHD1.

In addition to nonsyndromic hearing loss, an enrichment of LOXHDl variants in patients affected by Fuchs endothelial corneal dystrophy (FECD) has been observed in one study, suggesting that LOXHD1 could be relevant to the FECD pathogenesis (Riazuddin et al. 2012). Conversely, other studies have detected no association between LOXHDI variants and FECD in the HL patients and their relatives (Bai et al. 2020; Wesdorp et al. 2018). Therefore, the link between FECD and LOXHD1 is yet unconfirmed, with a need for further evidence. We did not observe signs of corneal dystrophy in any of the eye examined dogs either homozygous or heterozygous for the LOXHD1 variant. However, we cannot exclude the possibility of later onset clinical signs.

In conclusion, we describe a rare novel missense variant in $L O X H D I$ associated with canine autosomal recessive nonsyndromic hearing loss, providing a new animal model for human hearing disorders. The variant was observed to be specific to the Rottweiler breed. The affected breed will benefit from a genetic test to eradicate the hearing impairment from the population.
Supplementary Information The online version contains supplementary material available at https://doi.org/10.1007/s00439-021-02286-z.

Acknowledgements We would like to acknowledge the Biomedicum Functional Genomics Unit (FuGU) and the Institute for Molecular Medicine Finland core facility (FIMM) at the University of Helsinki for sequencing services, as well as the IT Center for Science Ltd. (CSC, Finland) for providing high-performance computing infrastructure. Sini Karjalainen and Ileana Quintero are thanked for their skillful technical help. We thank the Dog Biomedical Variant Database Consortium (DBVDC), Gustavo Aguirre, Catherine André, Danika Bannasch, Doreen Becker, Cord Drögemüller, Kari Ekenstedt, Kiterie Faller, Oliver Forman, Steve Friedenberg, Eva Furrow, Urs Giger, Christophe Hitte, Marjo K Hytönen, Vidhya Jagannathan, Frode Lingaas, Tosso Leeb, Hannes Lohi, Cathryn Mellersh, James R Mickelson, Leonardo Murgiano, Anita Oberbauer, Sheila Schmutz, Jeffrey J Schoenebeck, Kim Summers, Frank van Steenbeek and Claire Wade for sharing the control genome sequence data from dogs and wolves. We are grateful to the more than 1000 Banfield clinicians who diligently and consistently recorded their observations in a medical record during 2019-2021 years. Without that collective effort this work would not have been possible. We are grateful to all dog owners who have donated samples from their dogs for the study.

Author contributions Conceptualization: HL and MKH; design: MKH; investigation: MKH; formal analysis: MKH, JEN, MA, CAB-K, JD; writing — original draft: $\mathrm{MKH}, \mathrm{JEN}$; writing — editing and review: all; funding acquisition: HL.

Funding Open access funding provided by University of Helsinki including Helsinki University Central Hospital. This study was partially supported by the Jane and Aatos Erkko Foundation, the Academy of Finland, Helsinki Institute of Life Science, and Wisdom Health.

Availability of data and material Exome and whole genome sequencing data from three dogs have been published in the SRA under the BioProject accession number PRJNA702911 https://www.ncbi.nlm.nih. gov/sra/?term=PRJNA702911.

\section{Declarations}

Conflicts of interest JD and CAB-K are employees of Wisdom Health, and $\mathrm{HL}$ is a paid consultant to Genoscoper Laboratories Ltd, providing genetic tests for dogs.

Ethics approval Sample collection was ethically approved by the Animal Ethics Committee of State Provincial Office of Southern Finland (ESAVI/343/04.10. 07/2016 and ESAVI/25696/2020).

Consent to participate Written consent was given by the dogs' owners, who donated samples from their dogs.

Open Access This article is licensed under a Creative Commons Attribution 4.0 International License, which permits use, sharing, adaptation, distribution and reproduction in any medium or format, as long as you give appropriate credit to the original author(s) and the source, provide a link to the Creative Commons licence, and indicate if changes were made. The images or other third party material in this article are included in the article's Creative Commons licence, unless indicated otherwise in a credit line to the material. If material is not included in the article's Creative Commons licence and your intended use is not permitted by statutory regulation or exceeds the permitted use, you will 
need to obtain permission directly from the copyright holder. To view a copy of this licence, visit http://creativecommons.org/licenses/by/4.0/.

\section{References}

Adzhubei IA, Schmidt S, Peshkin L, Ramensky VE, Gerasimova A, Bork P, Kondrashov AS, Sunyaev SR (2010) A method and server for predicting damaging missense mutations. Nat Methods 7:248 249. https://doi.org/10.1038/nmeth0410-248

Arumilli M, Layer RM, Hytönen MK, Lohi H (2020) webGQT: a shiny server for genotype query tools for model-based variant filtering. Front Genet 11:152. https://doi.org/10.3389/fgene.2020.00152

Bai X, Zhang C, Zhang F, Xiao Y, Jin Y, Wang H, Xu L (2020) Five novel mutations in $L O X H D 1$ gene were identified to cause autosomal recessive nonsyndromic hearing loss in four Chinese families. Biomed Res Int 2020:1685974. https://doi.org/10.1155/2020/ 1685974

Bateman A, Sandford R (1999) The PLAT domain: a new piece in the PKD1 puzzle. Curr Biol 9:588

Broeckx BJ, Hitte C, Coopman F, Verhoeven GE, De Keulenaer S, De Meester E, Derrien T, Alfoldi J, Lindblad-Toh K, Bosmans T, Gielen I, Van Bree H, Van Ryssen B, Saunders JH, Van Nieuwerburgh F, Deforce D (2015) Improved canine exome designs, featuring ncRNAs and increased coverage of protein coding genes. Sci Rep 5:12810. https://doi.org/10.1038/srep12810

Buniello A, MacArthur JAL, Cerezo M, Harris LW, Hayhurst J, Malangone C, McMahon A, Morales J, Mountjoy E, Sollis E, Suveges D, Vrousgou O, Whetzel PL, Amode R, Guillen JA, Riat HS, Trevanion SJ, Hall P, Junkins H, Flicek P, Burdett T, Hindorff LA, Cunningham F, Parkinson H (2019) The NHGRI-EBI GWAS catalog of published genome-wide association studies, targeted arrays and summary statistics 2019. Nucleic Acids Res 47:D1005-D1012. https://doi.org/10.1093/nar/gky1120

Chang CC, Chow CC, Tellier LC, Vattikuti S, Purcell SM, Lee JJ (2015) Second-generation PLINK: rising to the challenge of larger and richer datasets. Gigascience 4:7-8. https://doi.org/10.1186/ s13742-015-0047-8

Chiang C, Layer RM, Faust GG, Lindberg MR, Rose DB, Garrison EP, Marth GT, Quinlan AR, Hall IM (2015) SpeedSeq: ultrafast personal genome analysis and interpretation. Nat Methods 12:966-968. https://doi.org/10.1038/nmeth.3505

Choi Y, Chan AP (2015) PROVEAN web server: a tool to predict the functional effect of amino acid substitutions and indels. Bioinformatics 31:2745-2747. https://doi.org/10.1093/bioinformatics/ btv195

Choi Y, Sims GE, Murphy S, Miller JR, Chan AP (2012) Predicting the functional effect of amino acid substitutions and indels. PLoS ONE 7:e46688. https://doi.org/10.1371/journal.pone.0046688

Comito B, Knowles KE, Strain GM (2012) Congenital deafness in Jack Russell terriers: prevalence and association with phenotype. Vet J 193:404-407. https://doi.org/10.1016/j.tvj1.2012.02.018

Coppens AG, Kiss R, Heizmann CW, Deltenre P, Poncelet L (2001) An original inner ear neuroepithelial degeneration in a deaf Rottweiler puppy. Hear Res 161:65-71

De Risio L, Freeman J, Lewis T (2016) Prevalence, heritability and genetic correlations of congenital sensorineural deafness and coat pigmentation phenotype in the English bull terrier. BMC Vet Res 12:146-156. https://doi.org/10.1186/s12917-016-0777-6

Edvardson S, Jalas C, Shaag A, Zenvirt S, Landau C, Lerer I, Elpeleg O (2011) A deleterious mutation in the LOXHD1 gene causes autosomal recessive hearing loss in Ashkenazi Jews. Am J Med Genet A 155A:1170-1172. https://doi.org/10.1002/ajmg.a.33972

Gardner EJ, Lam VK, Harris DN, Chuang NT, Scott EC, Pittard WS, Mills RE, 1000 Genomes Project Consortium, Devine SE (2017)
The mobile element locator tool (MELT): population-scale mobile element discovery and biology. Genome Res 27:1916-1929. https://doi.org/10.1101/gr.218032.116

Golosova O, Henderson R, Vaskin Y, Gabrielian A, Grekhov G, Nagarajan V, Oler AJ, Quinones M, Hurt D, Fursov M, Huyen Y (2014) Unipro UGENE NGS pipelines and components for variant calling, RNA-seq and ChIP-seq data analyses. PeerJ 2:e644. https:// doi.org/10.7717/peerj.644

Grillet N, Schwander M, Hildebrand MS, Sczaniecka A, Kolatkar A, Velasco J, Webster JA, Kahrizi K, Najmabadi H, Kimberling WJ, Stephan D, Bahlo M, Wiltshire T, Tarantino LM, Kuhn P, Smith RJ, Muller U (2009) Mutations in LOXHD1, an evolutionarily conserved stereociliary protein, disrupt hair cell function in mice and cause progressive hearing loss in humans. Am J Hum Genet 85:328-337. https://doi.org/10.1016/j.ajhg.2009.07.017

Guevar J, Olby NJ, Meurs KM, Yost O, Friedenberg SG (2018) Deafness and vestibular dysfunction in a Doberman Pinscher puppy associated with a mutation in the $P T P R Q$ gene. J Vet Intern Med 32:665-669. https://doi.org/10.1111/jvim.15060

Hytönen MK, Arumilli M, Sarkiala E, Nieminen P, Lohi H (2019) Canine models of human amelogenesis imperfecta: identification of novel recessive ENAM and ACP4 variants. Hum Genet 138:525-533. https://doi.org/10.1007/s00439-019-01997-8

Johnson M, Zaretskaya I, Raytselis Y, Merezhuk Y, McGinnis S, Madden TL (2008) NCBI BLAST: a better web interface. Nucleic Acids Res 36:5. https://doi.org/10.1093/nar/gkn201

Jurka J, Kapitonov VV, Pavlicek A, Klonowski P, Kohany O, Walichiewicz J (2005) Repbase update, a database of eukaryotic repetitive elements. Cytogenet Genome Res 110:462-467

Karlsson EK, Baranowska I, Wade CM, Salmon Hillbertz NH, Zody MC, Anderson N, Biagi TM, Patterson N, Pielberg GR, Kulbokas EJ, Comstock KE, Keller ET, Mesirov JP, von Euler H, Kämpe O, Hedhammar A, Lander ES, Andersson G, Andersson L, Lindblad-Toh K (2007) Efficient mapping of mendelian traits in dogs through genome-wide association. Nat Genet 39:1321-1328. https://doi.org/10.1038/ng.2007.10

Lindblad-Toh K, Wade CM, Mikkelsen TS, Karlsson EK, Jaffe DB, Kamal M, Clamp M, Chang JL, Kulbokas EJ, Zody MC, Mauceli E, Xie X, Breen M, Wayne RK, Ostrander EA, Ponting CP, Galibert F, Smith DR, DeJong PJ, Kirkness E, Alvarez P, Biagi T, Brockman W, Butler J, Chin CW, Cook A, Cuff J, Daly MJ, DeCaprio D, Gnerre S, Grabherr M, Kellis M, Kleber M, Bardeleben C, Goodstadt L, Heger A, Hitte C, Kim L, Koepfli KP, Parker HG, Pollinger JP, Searle SM, Sutter NB, Thomas R, Webber C, Baldwin J, Abebe A, Abouelleil A, Aftuck L, AitZahra M, Aldredge T, Allen N, An P, Anderson S, Antoine C, Arachchi H, Aslam A, Ayotte L, Bachantsang P, Barry A, Bayul T, Benamara M, Berlin A, Bessette D, Blitshteyn B, Bloom T, Blye J, Boguslavskiy L, Bonnet C, Boukhgalter B, Brown A, Cahill P, Calixte N, Camarata J, Cheshatsang Y, Chu J, Citroen M, Collymore A, Cooke P, Dawoe T, Daza R, Decktor K, DeGray S, Dhargay N, Dooley K, Dooley K, Dorje P, Dorjee K, Dorris L, Duffey N, Dupes A, Egbiremolen O, Elong R, Falk J, Farina A, Faro S, Ferguson D, Ferreira P, Fisher S, FitzGerald M, Foley K, Foley C, Franke A, Friedrich D, Gage D, Garber M, Gearin G, Giannoukos G, Goode T, Goyette A, Graham J, Grandbois E, Gyaltsen K, Hafez N, Hagopian D, Hagos B, Hall J, Healy C, Hegarty R, Honan T, Horn A, Houde N, Hughes L, Hunnicutt L, Husby M, Jester B, Jones C, Kamat A, Kanga B, Kells C, Khazanovich D, Kieu AC, Kisner P, Kumar M, Lance K, Landers T, Lara M, Lee W, Leger JP, Lennon N, Leuper L, LeVine S, Liu J, Liu X, Lokyitsang Y, Lokyitsang T, Lui A, Macdonald J, Major J, Marabella R, Maru K, Matthews C, McDonough S, Mehta T, Meldrim J, Melnikov A, Meneus L, Mihalev A, Mihova T, Miller K, Mittelman R, Mlenga V, Mulrain L, Munson G, Navidi A, Naylor J, Nguyen T, Nguyen N, Nguyen C, Nguyen T, Nicol R, 
Norbu N, Norbu C, Novod N, Nyima T, Olandt P, O’Neill B, O’Neill K, Osman S, Oyono L, Patti C, Perrin D, Phunkhang P, Pierre F, Priest M, Rachupka A, Raghuraman S, Rameau R, Ray V, Raymond C, Rege F, Rise C, Rogers J, Rogov P, Sahalie J, Settipalli S, Sharpe T, Shea T, Sheehan M, Sherpa N, Shi J, Shih D, Sloan J, Smith C, Sparrow T, Stalker J, Stange-Thomann N, Stavropoulos S, Stone C, Stone S, Sykes S, Tchuinga P, Tenzing P, Tesfaye S, Thoulutsang D, Thoulutsang Y, Topham K, Topping I, Tsamla T, Vassiliev H, Venkataraman V, Vo A, Wangchuk T, Wangdi T, Weiand M, Wilkinson J, Wilson A, Yadav S, Yang S, Yang X, Young G, Yu Q, Zainoun J, Zembek L, Zimmer A, Lander ES (2005) Genome sequence, comparative analysis and haplotype structure of the domestic dog. Nature 438:803-819. https://doi.org/10.1038/nature04338

Maekawa K, Nishio SY, Abe S, Goto SI, Honkura Y, Iwasaki S, Kanda Y, Kobayashi Y, Oka SI, Okami M, Oshikawa C, Sakuma N, Sano H, Shirakura M, Uehara N, Usami SI (2019) Mutational spectrum and clinical features of patients with $L O X H D 1$ variants identified in an 8074 hearing loss patient cohort. Genes (Basel). https://doi. org/10.3390/genes10100735.E735

McKenna A, Hanna M, Banks E, Sivachenko A, Cibulskis K, Kernytsky A, Garimella K, Altshuler D, Gabriel S, Daly M, DePristo MA (2010) The genome analysis toolkit: a mapreduce framework for analyzing next-generation DNA sequencing data. Genome Res 20:1297-1303. https://doi.org/10.1101/gr.107524.110

Meyermans R, Gorssen W, Buys N, Janssens S (2020) How to study runs of homozygosity using PLINK? A guide for analyzing medium density SNP data in livestock and pet species. BMC Genomics 21:94-x. https://doi.org/10.1186/s12864-020-6463-x

Mori K, Moteki H, Kobayashi Y, Azaiez H, Booth KT, Nishio SY, Sato H, Smith RJ, Usami S (2015) Mutations in LOXHD1 gene cause various types and severities of hearing loss. Ann Otol Rhinol Laryngol 124(Suppl 1):135S-41S. https://doi.org/10.1177/ 0003489415574067

Morton CC, Nance WE (2006) Newborn hearing screening-a silent revolution. N Engl J Med 354:2151-2164

Okonechnikov K, Golosova O, Fursov M, UGENE team (2012) Unipro UGENE: a unified bioinformatics toolkit. Bioinformatics 28:1166-1167. https://doi.org/10.1093/bioinformatics/bts091

Papadopoulos JS, Agarwala R (2007) COBALT: constraint-based alignment tool for multiple protein sequences. Bioinformatics 23:1073-1079

Platt S, Freeman J, di Stefani A, Wieczorek L, Henley W (2006) Prevalence of unilateral and bilateral deafness in border collies and association with phenotype. J Vet Intern Med 20:1355-1362. https://doi.org/10.1892/0891-6640(2006)20[1355:pouabd]2.0. co;2

Plevova P, Paprskarova M, Tvrda P, Turska P, Slavkovsky R, Mrazkova E (2017) STRC deletion is a frequent cause of slight to moderate congenital hearing impairment in the Czech Republic. Otol Neurotol 38:e393-e400. https://doi.org/10.1097/MAO.0000000000 001571
Purfield DC, Berry DP, McParland S, Bradley DG (2012) Runs of homozygosity and population history in cattle. BMC Genet 13:70. https://doi.org/10.1186/1471-2156-13-70.

Rausch T, Zichner T, Schlattl A, Stutz AM, Benes V, Korbel JO (2012) DELLY: structural variant discovery by integrated paired-end and split-read analysis. Bioinformatics 28:i333-i339. https://doi.org/ 10.1093/bioinformatics/bts378

Riazuddin SA, Parker DS, McGlumphy EJ, Oh EC, Iliff BW, Schmedt T, Jurkunas U, Schleif R, Katsanis N, Gottsch JD (2012) Mutations in $L O X H D 1$, a recessive-deafness locus, cause dominant late-onset Fuchs corneal dystrophy. Am J Hum Genet 90:533-539. https://doi.org/10.1016/j.ajhg.2012.01.013

Rose R, Golosova O, Sukhomlinov D, Tiunov A, Prosperi M (2019) Flexible design of multiple metagenomics classification pipelines with UGENE. Bioinformatics 35:1963-1965. https://doi.org/10. 1093/bioinformatics/bty901

Shen N, Wang T, Li D, Liu A, Lu Y (2019) Whole-exome sequencing identifies a novel missense variant within $L O X H D 1$ causing rare hearing loss in a Chinese family. BMC Med Genet 20:30-2. https://doi.org/10.1186/s12881-019-0758-2

Strain GM (1996) Aetiology, prevalence and diagnosis of deafness in dogs and cats. Br Vet J 152:17-36

Strain GM (2004) Deafness prevalence and pigmentation and gender associations in dog breeds at risk. Vet J 167:23-32

Strain GM (2015) The genetics of deafness in domestic animals. Front Vet Sci 2:29. https://doi.org/10.3389/fvets.2015.00029

Webb AA, Ruhe AL, Neff MW (2019) A missense mutation in MYO7A is associated with bilateral deafness and vestibular dysfunction in the Doberman pinscher breed. Can J Vet Res 83:142-148

Wesdorp M, Schreur V, Beynon AJ, Oostrik J, van de Kamp JM, Elting MW, van den Boogaard MH, Feenstra I, Admiraal RJC, Kunst HPM, Hoyng CB, Kremer H, Yntema HG, Pennings RJE, Schraders M (2018) Further audiovestibular characterization of DFNB77, caused by deleterious variants in $L O X H D 1$, and investigation into the involvement of Fuchs corneal dystrophy. Clin Genet 94:221-231. https://doi.org/10.1111/cge.13368

Yokoyama JS, Lam ET, Ruhe AL, Erdman CA, Robertson KR, Webb AA, Williams DC, Chang ML, Hytönen MK, Lohi H, Hamilton SP, Neff MW (2012) Variation in genes related to cochlear biology is strongly associated with adult-onset deafness in border collies. PLoS Genet 8:e1002898. https://doi.org/10.1371/journal.pgen. 1002898

Zhang C, Hao S, Liu Y, Zhou B, Liu F, Zheng L, Ma P, Liu Q, Lin X, Yan Y, Zhang Q (2019) A novel LOXHDl variant in a Chinese couple with hearing loss. J Int Med Res 47:6082-6090. https:// doi.org/10.1177/0300060519884197

Publisher's Note Springer Nature remains neutral with regard to jurisdictional claims in published maps and institutional affiliations. 